\title{
MSSM inflaton: SUSY dark matter and LHC
}

\author{
A. Mazumdar \\ Physics Department, Lancaster University, Lancaster, LAI 4YB, UK \\ Niels Bohr Institute, Copenhagen University, Blegdamsvej-17, DK-2100, Denmark
}

\begin{abstract}
In this talk we will discuss how inflation can be embedded within a minimal extension of the Standard Model where the inflaton carries the Standard Model charges. There is no need of an ad-hoc scalar field to be introduced in order to explain the temperature anisotropy of the cosmic microwave background radiation, all the ingredients are present within a minimal supersymmetric Standard Model. For the first time inflaton properties can be directly linked to the particle phenomenology, dark matter, and the baryons of the Standard Model.
\end{abstract}

Keywords: Inflation, baryons, dark matter

PACS: $98.80 \mathrm{Cq}$

Inflation has been extremely successful in explaining the temperature anisotropy of the observed comsic microwave background radiation by generating almost scale invariant density perturbations [1]. It has been known that inflation can be driven by a dynamical scalar field known as the inflaton, an order parameter, which could either be fundamental or composite. Particularly, if the inflaton rolls very slowly on a sufficiently flat potential such that the potential energy density dominates over the kinetic term, then all the successes of inflation can be met, i.e. dynamical explanation of the homogeneity and the isotropy of the universe on very large scales, nearly Gaussian density perturbations, etc.

Inspite of the impressive list of achievements, it has been proven hard to embed inflation in a fundamental theory which could also be testable in a laboratory. In past one has always relied on scalar fields which are absolute gauge singlets possibly residing in a hidden sector or a secluded sector with an unknown couplings to the SM gauge group. By definition, an absolute gauge singlet does not carry any charge what so-ever be the case, therefore, the masses, couplings and interactions are not generally tied to any fundamental theory or any symmetry. Such gauge singlets are used ubiquitously by model builders to obtain a desired potential and interactions almost at a free will in order to explain the current CMB data.

Very recently some of these questions have been addressed in a low energy field theory setup, which explains (for a review see [2]): a) the origin of inflation, b) the fundamental interactions of an inflaton, c) how the inflaton creates Standard Model baryons and cold dark matter?, and d) how can we test the inflaton in a laboratory?

For the first time we have been successful in constructing an inflaton which carries the Standard Model (SM) charges and embedded within supersymmetry(SUSY) [3, 4, 5, 6]. The SUSY provides the scalar fields (partners of the SM fermions and gauge bosons) and the stability of the flatness of the inflaton potential. Since the inflaton carries the SM charges, it decays only into the SM particles and SUSY particles, i.e. quarks, squarks, leptons, sleptons, etc. Within a minimal supersymmetric Standard Model (MSSM) we 


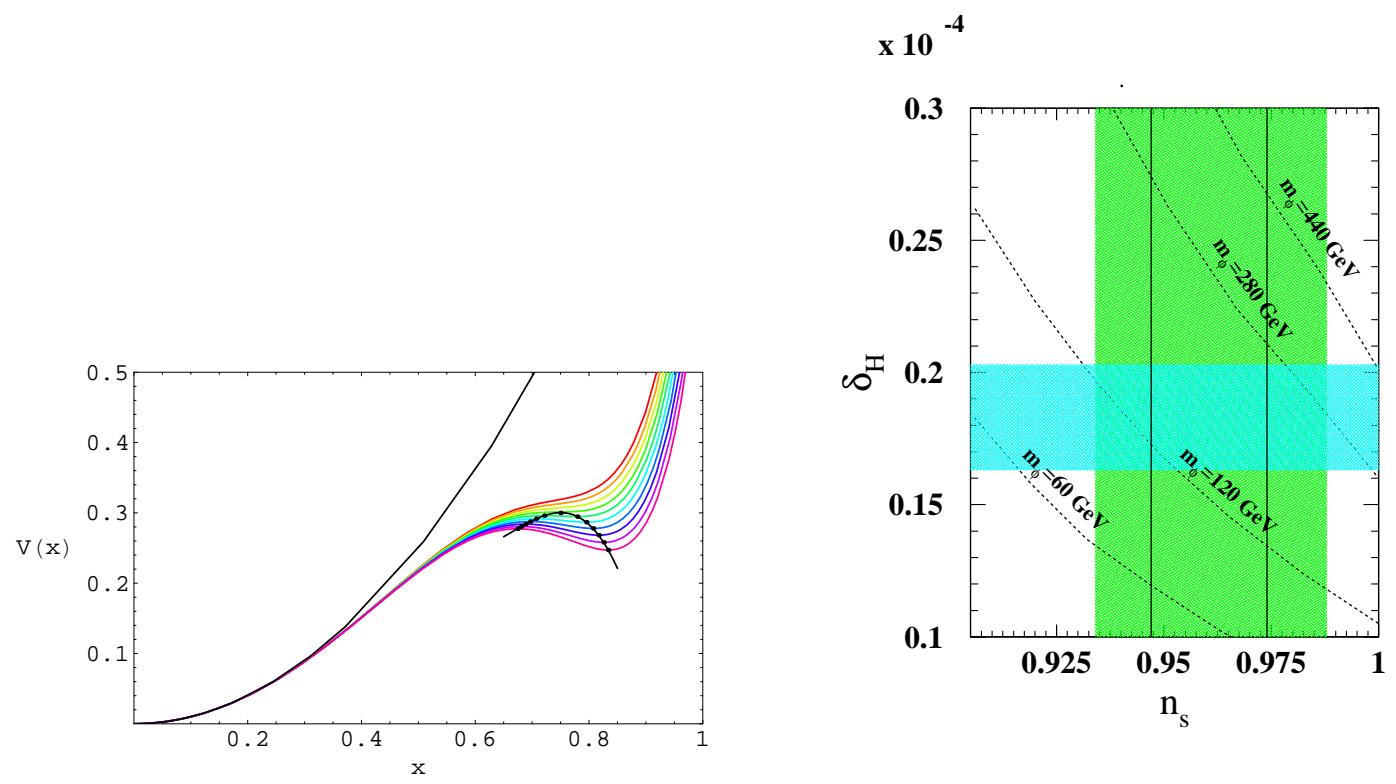

FIGURE 1. The shape of the potential on the left hand side. On the right hand side, we have shown the constraints on the mass of the inflaton, $m_{\phi}\left(\phi_{0}=10^{14} \mathrm{GeV}\right)$, from the amplitude, $\delta_{H}$, and tilt, $n_{s}$, of the power spectrum. Note that the inflaton is made up of either $u d d$ or LLe, see Ref. [8].

know all the relativistic species and therefore we can trace back thermal history of the universe accurately above the electroweak scale. If the lightest supersymmetric particle (LSP) is stable due to R-parity, we naturally obtain cold dark matter after the MSSM inflation [7].

Let us now mention the main features of MSSM flat direction inflation [3, 4]. There are only two flat directions, $u d d$ and $L L e$, which are lifted by higher order superpotential terms of the following form [9, 10]:

$$
W \supset \frac{\lambda}{6} \frac{\Phi^{6}}{M_{\mathrm{P}}^{3}}
$$

where $\lambda \sim \mathscr{O}(1)$, rest of the flat directions within MSSM are lifted by hybrid superpotential terms, i.e. $W \supset\left(\Phi^{n-1} \Psi\right) / M_{\mathrm{P}}^{n-3}$. Such terms yield no non-renormalizable A-term in the potential, which is relevant for our discussion below. The scalar component of $\Phi$ superfield, denoted by $\phi$, is given by

$$
\phi=\frac{u+d+d}{\sqrt{3}} \quad, \quad \phi=\frac{L+L+e}{\sqrt{3}},
$$

for the $u d d$ and $L L e$ flat directions respectively.

Writing the complex scalar field $\Phi$ in terms of radial and angular components $\Phi=$ $\phi \exp [i \theta]$, the scalar potential along the radial direction $\phi$ is found to be [3, 4]

$$
V(\phi)=\frac{1}{2} m_{\phi}^{2} \phi^{2}-A \frac{\lambda \phi^{6}}{6 M_{\mathrm{P}}^{6}}+\lambda^{2} \frac{\phi^{10}}{M_{\mathrm{P}}^{6}},
$$

where $m_{\phi}$ and $A$ are the soft breaking mass and the $A$-term respectively. We have already minimized the potential along the angular direction $\theta$. Note that $A$ is a positive quantity 
as we can absorb its phase by a redefinition of $\theta$. Provided that $\frac{A^{2}}{40 m_{\phi}^{2}} \equiv 1+4 \alpha^{2}$, where $\alpha^{2} \ll 1$, there exists a point of inflection in $V(\phi)[7,11]$

$$
\phi_{0}=\left(\frac{m_{\phi} M_{\mathrm{P}}^{3}}{\lambda \sqrt{10}}\right)^{1 / 4}+\mathscr{O}\left(\alpha^{2}\right), \quad V^{\prime \prime}\left(\phi_{0}\right)=0
$$

at which

$V\left(\phi_{0}\right)=\frac{4 m_{\phi}^{2} \phi_{0}^{2}}{15}+\mathscr{O}\left(\alpha^{2}\right), V^{\prime}\left(\phi_{0}\right)=4 \alpha^{2} m_{\phi}^{2} \phi_{0}+\mathscr{O}\left(\alpha^{4}\right), V^{\prime \prime \prime}\left(\phi_{0}\right)=\frac{32 m_{\phi}^{2}}{\phi_{0}}+\mathscr{O}\left(\alpha^{2}\right)$

From now on we only keep the leading order terms in all expressions. Note that in gravity mediated SUSY breaking, the $A$-term and the soft SUSY breaking mass are of the same order of magnitude as the gravitino mass, i.e. $m_{\phi} \sim A \sim m_{3 / 2} \sim(100 \mathrm{GeV}-$ $1 \mathrm{TeV})$. Therefore the above conditions can indeed be satisfied. We then have $\phi_{0} \sim$ $\mathscr{O}\left(10^{14} \mathrm{GeV}\right)$. Inflation occurs within an interval, $\left|\phi-\phi_{0}\right| \sim \frac{\phi_{0}^{3}}{60 M_{\mathrm{P}}^{2}}$, in the vicinity of the point of inflection, within which the slow roll parameters $\varepsilon \equiv\left(M_{\mathrm{P}}^{2} / 2\right)\left(V^{\prime} / V\right)^{2}$ and $\eta \equiv M_{\mathrm{P}}^{2}\left(V^{\prime \prime} / V\right)$ are smaller than 1 . The Hubble expansion rate during inflation is given by

$$
H_{\mathrm{MSSM}} \simeq \frac{1}{\sqrt{45}} \frac{m_{\phi} \phi_{0}}{M_{\mathrm{P}}} \sim(100 \mathrm{MeV}-1 \mathrm{GeV}) .
$$

The amplitude of density perturbations $\delta_{H}$ and the scalar spectral index $n_{S}$ are given by [4, 7, 8,11$]$ :

$$
\delta_{H}=\frac{8}{\sqrt{5} \pi} \frac{m_{\phi} M_{\mathrm{P}}}{\phi_{0}^{2}} \frac{1}{\Delta^{2}} \sin ^{2}\left[\mathscr{N}_{\mathrm{COBE}} \sqrt{\Delta^{2}}\right], \quad n_{s}=1-4 \sqrt{\Delta^{2}} \cot \left[\mathscr{N}_{\mathrm{COBE}} \sqrt{\Delta^{2}}\right],
$$

where

$$
\Delta^{2} \equiv 900 \alpha^{2} \mathscr{N}_{\operatorname{COBE}}^{-2}\left(\frac{M_{\mathrm{P}}}{\phi_{0}}\right)^{4} .
$$

$\mathscr{N}_{\text {COBE }}$ is the number of e-foldings between the time when the observationally relevant perturbations are generated till the end of inflation and follows: $\mathscr{N}_{\mathrm{COBE}} \simeq 66.9+$ $(1 / 4) \ln \left(V\left(\phi_{0}\right) / M_{\mathrm{P}}^{4}\right) \sim 50$ [12]. We note that reheating after MSSM inflation is very fast, due to gauge couplings of the inflaton to gauge/gaugino fields, and results in a radiationdominated universe within few Hubble times after the end of inflation [4, 13].

A remarkable property of MSSM inflation, which is due to inflation occurring near a point of inflection, is that it can give rise to a wide range of scalar spectral index. This is in clear distinction with other models (for example, chaotic inflation, hybrid inflation natural inflation, etc.) and makes the model very robust. Indeed it can yield a spectral index within the whole $2 \sigma$ allowed range by 5 -year WMAP data $0.934 \leq n_{s} \leq 0.988$ [1]. This happens for

$$
0 \leq \Delta^{2} \leq \frac{\pi^{2}}{4 \mathscr{N}_{\mathrm{COBE}}^{2}}
$$


In Fig. (1), we show $\delta_{H}$ as a function of $n_{s}$ for different values of $m_{\phi}\left(\phi_{0}\right)$. The horizontal blue band shows the $2 \sigma$ experimental band for $\delta_{H}$. The vertical green shaded region is the $2 \sigma$ experimental band for $n_{s}$. The region enclosed by solid lines shows the $1 \sigma$ experimental allowed region. We find that smaller values of $m_{\phi}$ are preferred for smaller values of $n_{s}$. We also find that the allowed range of $m_{\phi}$ is $90-330 \mathrm{GeV}$ for the experimental ranges of $n_{s}$ and $\delta_{H}$. This figure is drawn for $\lambda \simeq 1$, which is natural in the context of effective field theory (unless it is suppressed due to some symmetry). Smaller values of $\lambda$ will lead to an increase in $m_{\phi}$ [7].

Initial condition for inflation has been studied in Ref. [14] and in Ref. [8], we showed that MSSM inflaton is always driven to the point of inflection if there is an earlier false vacuum phase of inflation. In this respect, although inflation occurs at a low scale, the initial conditions are natural once the MSSM inflation is preceded by a high scale inflation, i.e. $H_{\text {inf }} \geq H_{\text {MSSM }}$. We also showed how to realize such a false vacuum inflation within MSSM. Recently we have also shown that the MSSM inflaton will fragment after inflation, and the process of fragmentation will leaves its imprints in the gravity waves, with a detectable frequency and amplitude which matches with that of LISA and LIGO-III experiments [15].

Since $m_{\phi}$ is related to the scalar masses, sleptons (LLe direction) and squarks (udd direction), the bound on $m_{\phi}$ will be translated into the bounds on these scalar masses which are expressed in terms of the model parameters [4]. The models of mSUGRA depend only on four parameters and one sign. These are $m_{0}$ (the universal scalar soft breaking mass at the GUT scale $M_{\mathrm{G}}$ ); $m_{1 / 2}$ (the universal gaugino soft breaking mass at $M_{\mathrm{G}}$ ); $A_{0}$ (the universal trilinear soft breaking mass at $M_{\mathrm{G}}$ ); $\tan \beta=\left\langle H_{2}\right\rangle\left\langle H_{1}\right\rangle$ at the electroweak scale (where $H_{2}$ gives rise to $u$ quark masses and $H_{1}$ to $d$ quark and lepton masses); and the sign of $\mu$, the Higgs mixing parameter in the superpotential $\left(W_{\mu}=\mu H_{1} H_{2}\right)$. Unification of gauge couplings within supersymmetry suggests that $M_{\mathrm{G}} \simeq 2 \times 10^{16} \mathrm{GeV}$. The model parameters are already significantly constrained by different experimental results. Most important constraints are: The light Higgs mass bound of $M_{h^{0}}>114.0 \mathrm{GeV}$ from LEP [16]. The $b \rightarrow s \gamma$ branching ratio: $2.2 \times 10^{-4}<$ $\mathscr{B}\left(B \rightarrow X_{s} \gamma\right)<4.5 \times 10^{-4}$ [17]. In mSUGRA the $\tilde{\chi}_{1}^{0}$ is the candidate for CDM. The $2 \sigma$ bound from the WMAP gives a relic density bound for CDM to be $0.095<\Omega_{\mathrm{CDM}} h^{2}<$ 0.129 [1]. The bound on the lightest chargino mass of $M_{\tilde{\chi}_{1}^{ \pm}}>104 \mathrm{GeV}$ from LEP [18]. The possible $3.3 \sigma$ deviation (using $e^{+} e^{-}$data to calculate the leading order hadronic contribution)from the SM expectation of the anomalous muon magnetic moment from the muon $g-2$ collaboration [19].

The allowed mSUGRA parameter space, at present, has mostly three distinct regions: (i) the stau-neutralino $\left(\tilde{\tau}_{1}-\tilde{\chi}_{0}^{1}\right)$, coannihilation region where $\tilde{\chi}_{0}^{1}$ is the lightest SUSY particle (LSP), (ii) the $\tilde{\chi}_{0}^{1}$ having a dominant Higgsino component (focus point) and (iii) the scalar Higgs $\left(A^{0}, H^{0}\right)$ annihilation funnel $\left(2 M_{\tilde{\chi}_{0}^{1}} \simeq M_{A^{0}, H^{0}}\right)$ [20]. These three regions have been selected out by the CDM constraint. There stills exists a bulk region where none of these above properties is observed, but this region is now very small due to the existence of other experimental bounds. After considering all these bounds we will show that there exists an interesting overlap between the constraints from inflation and the CDM abundance. 

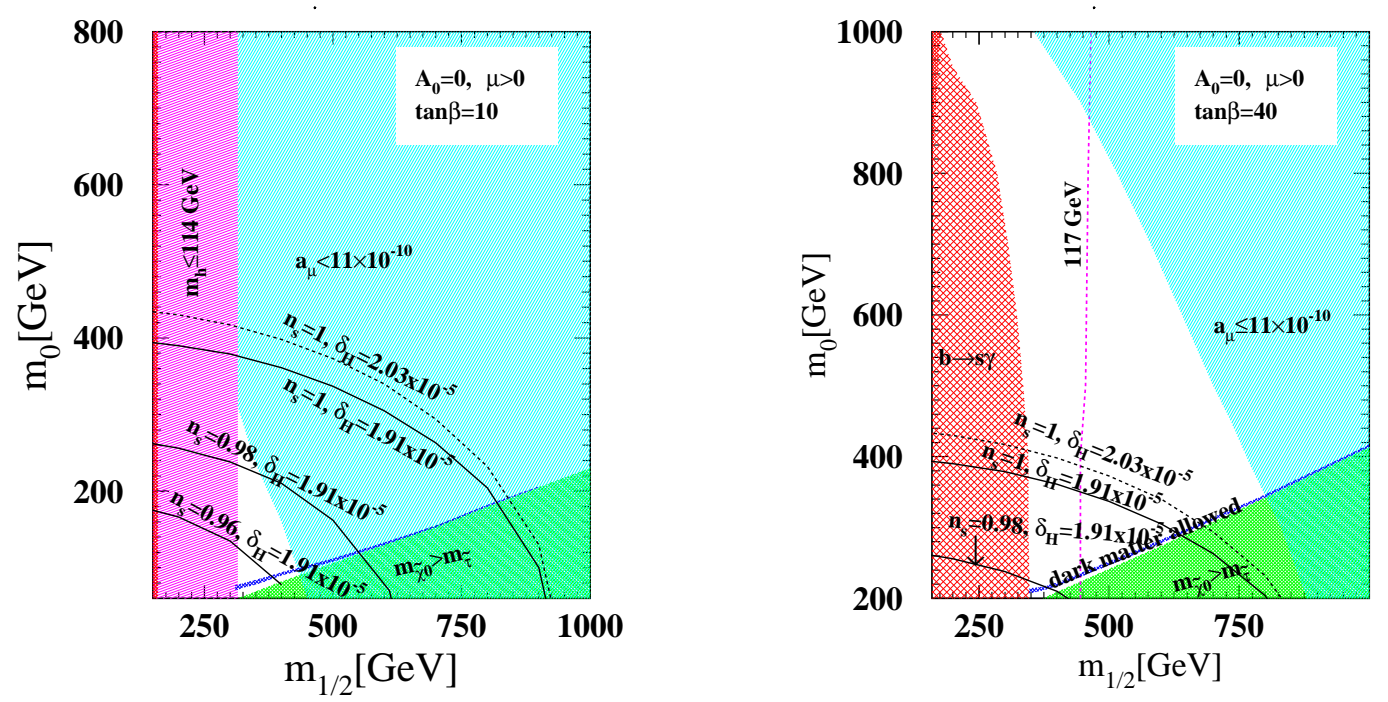

FIGURE 2. The contours for different values of $n_{s}$ and $\delta_{H}$ are shown in the $m_{0}-m_{1 / 2}$ plane for $\tan \beta=10$ and $\tan \beta=40$. We used $\lambda=1$ for the contours. We show the dark matter allowed region narrow blue corridor, (g-2) $\mu$ region (light blue) for $a_{\mu} \leq 11 \times 10^{-8}$, Higgs mass $\leq 114 \mathrm{GeV}$ (pink region) and LEPII bounds on SUSY masses (red). We also show the the dark matter detection rate by vertical blue lines. In the right hand panel, we $b \rightarrow s \gamma$ allowed region (brick).

We calculate $m_{\phi}$ at $\phi_{0}$ and $\phi_{0}$ is $10^{14} \mathrm{GeV}$ which is two orders of magnitude below the GUT scale. From this $m_{\phi}$, we determine $m_{0}$ and $m_{1 / 2}$ by solving the RGEs for fixed values of $A_{0}$ and $\tan \beta$. After we determine $m_{0}$ and $m_{1 / 2}$ from $m_{\phi}$, we can determine the allowed values of $m_{\phi}$ from the experimental bounds on the mSUGRA parameters space. In order to obtain the constraint on the mSUGRA parameter space, we calculate the SUSY particle masses by solving the RGEs at the weak scale using four parameters of the mSUGRA model and then use these masses to calculate Higgs mass, $B R[b \rightarrow s \gamma]$, dark matter content etc.

We show that the mSUGRA parameter space in Figs. (2) for $\tan \beta=10$ and 40 with the $u d d$ flat direction using $\lambda=1$. In the figures, we show contours correspond to $n_{s}=1$ for the maximum value of $\delta_{H}=2.03 \times 10^{-5}$ (at $2 \sigma$ level) and $n_{s}=1.0,0.98,0.96$ for $\delta_{H}=1.91 \times 10^{-5}$. The constraints on the parameter space arising from the inflation appearing to be consistent with the constraints arising from the dark matter content of the universe and other experimental results. We find that $\tan \beta$ needs to be smaller to allow for smaller values of $n_{s}<1$. It is also interesting to note that the allowed region of $m_{\phi}$, as required by the inflation data for $\lambda=1$ lies in the stau-neutralino coannihilation region which requires smaller values of the SUSY particle masses. The SUSY particles in this parameter space are, therefore, within the reach of the LHC very quickly. The detection of the region at the LHC has been considered in refs [21]. From the figures, one can also find that as $\tan \beta$ increases, the inflation data along with the dark matter, rare decay and Higgs mass constraint allow smaller ranges of $m_{1 / 2}$. For example, the allowed ranges of gluino masses are $765 \mathrm{GeV}-2.1 \mathrm{TeV}$ and $900 \mathrm{GeV}-1.7 \mathrm{TeV}$ for $\tan \beta=10$ and 40 respectively.

There are other relevant cases which we have not discussed here, we refer the readers to Ref. [7]. The dark matter abundance has also been studied when the inflaton is a gauge 
invariant combination of $\mathrm{NH}_{u} L$, first studied in Ref. [5], and the dark mater analysis was done in Ref. [22].

To summarize our analysis provides an example of a Standard Model gauge invariant inflaton giving rise to a successful inflation and explains the neutralino CDM abundance, which is in agreement with the present cosmological observations. Moreover this is the first example where the ingredients of a primordial inflation can be put onto test in a laboratory physics such as in the case of LHC.

\section{ACKNOWLEDGMENTS}

A.M is thankful to the organizers for their kind invitation. The author is also thankful to R. Allahverdi, B. Dutta, K. Enqvist, J. Garcia-Bellido and A. Jokinen and A. Kusenko for helpful discussions.

\section{REFERENCES}

1. D.N. Spergel, et.al., astro-ph/0603449.

2. A. Mazumdar,arXiv:0707.3350 [hep-ph].

3. R. Allahverdi, K. Enqvist, J. Garcia-Bellido and A. Mazumdar, Phys. Rev. Lett. 97, 191304 (2006)

4. R. Allahverdi, K. Enqvist, J. Garcia-Bellido, A. Jokinen and A. Mazumdar, JCAP 0706 (2007) 019.

5. R. Allahverdi, A. Kusenko and A. Mazumdar, JCAP 0707, 018 (2007).

6. R. Allahverdi, A. Jokinen and A. Mazumdar, arXiv:hep-ph/0610243.

7. R. Allahverdi, B. Dutta and A. Mazumdar, Phys. Rev. D 75, 075018 (2007).

8. R. Allahverdi, B. Dutta and A. Mazumdar,arXiv:0806.4557 [hep-ph].

9. For reviews, see K. Enqvist and A. Mazumdar, Phys. Rept. 380, 99 (2003); M. Dine and A. Kusenko, Rev. Mod. Phys. 76, 1 (2004).

10. T. Gherghetta, C. Kolda and S. P. Martin, Nucl. Phys. B 468, 37 (1996); M. Dine, L. Randall and S. Thomas, Phys. Rev. Lett. 75, 398 (1995); M. Dine, L. Randall and S. Thomas, Nucl. Phys. B 458, 291 (1996).

11. R. Allahverdi and A. Mazumdar, arXiv:hep-ph/0610069 J . C. Bueno Sanchez, K. Dimopoulos and D. H. Lyth, JCAP 0701, 015 (2007)

12. C. P. Burgess, R. Easther, A. Mazumdar, D. F. Mota and T. Multamaki, JHEP 0505, 067 (2005).

13. R. Allahverdi and A. Mazumdar, JCAP 0610, 008 (2006); JCAP 0708, 023 (2007).

14. R. Allahverdi, A. R. Frey and A. Mazumdar, Phys. Rev. D 76, 026001 (2007).

15. A. Kusenko and A. Mazumdar, Phys. Rev. Lett. 101, 211301 (2008).

16. ALEPH, DELPHI, L3, OPAL Collaborations, G. Abbiendi, et al. (The LEP Working Group for Higgs Boson Searches), Phys. Lett. B 565, 61 (2003).

17. M. Alam et al., Phys. Rev. Lett 74, 2885 (1995).

18. Particle Data Group, S. Eidelman et al., Phys. Lett. B 592, 1(2004).

19. Muon $g-2$ Collaboration, G. Bennett et al., Phys. Rev. Lett. 92, 161802 (2004); S. Eidelman, Talk at ICHEP 2006, Moscow, Russia.

20. J. Ellis, K. Olive, Y. Santoso, and V. Spanos, Phys. Lett. B 565, 176 (2003); R. Arnowitt, B. Dutta, and B. Hu, arXiv:hep-ph/0310103; H. Baer, C. Balazs, A. Belyaev, T. Krupovnickas, and X. Tata, JHEP 0306, 054 (2003); B. Lahanas and D.V. Nanopoulos, Phys. Lett. B 568, 55 (2003); U. Chattopadhyay, A. Corsetti, and P. Nath, Phys. Rev. Lett 68, 035005 (2003); E. Baltz and P. Gondolo, JHEP 0410, 052 (2004) 052; A. Djouadi, M. Drees and J. L. Kneur, JHEP 0603, 033 (2006); J. L. Feng and K. T. Matchev, Phys. Rev. Lett. 86, 3480 (2001).

21. R. Arnowitt et al., arXiv:hep-ph/0608193; R. Arnowitt, B. Dutta, T. Kamon, N. Kolev and D. Toback, Phys. Lett. B 639, 46 (2006)

22. R. Allahverdi, B. Dutta and A. Mazumdar, Phys. Rev. Lett. 99, 261301 (2007). 